\title{
ON THE STRUCTURE AND STABILITY OF MAGNETIC TOWER JETS
}

\author{
M. Huarte-Espinosa ${ }^{1}$, A. Frank ${ }^{1}$, E. G. Blackman ${ }^{1}$, A. Ciardi ${ }^{2,3}$, P. Hartigan ${ }^{4}$, S. V. Lebedev ${ }^{5}$, and J. P. Chittenden ${ }^{5}$ \\ ${ }^{1}$ Department of Physics and Astronomy, University of Rochester, 600 Wilson Boulevard, Rochester, NY 14627-0171, USA \\ ${ }^{2}$ LERMA, Université Pierre et Marie Curie, Observatoire de Paris, F-92195 Meudon, France \\ ${ }^{3}$ École Normale Supérieure, UMR 8112 CNRS, F-75231 Paris, France \\ ${ }^{4}$ Department of Physics and Astronomy, Rice University, 6100 S. Main, Houston, TX 77521-1892, USA \\ 5 The Blackett Laboratory, Imperial College London, SW7 2BW London, UK \\ Received 2012 April 1; accepted 2012 August 7; published 2012 September 5
}

\begin{abstract}
Modern theoretical models of astrophysical jets combine accretion, rotation, and magnetic fields to launch and collimate supersonic flows from a central source. Near the source, magnetic field strengths must be large enough to collimate the jet requiring that the Poynting flux exceeds the kinetic energy flux. The extent to which the Poynting flux dominates kinetic energy flux at large distances from the engine distinguishes two classes of models. In magneto-centrifugal launch models, magnetic fields dominate only at scales $\lesssim 100$ engine radii, after which the jets become hydrodynamically dominated (HD). By contrast, in Poynting flux dominated (PFD) magnetic tower models, the field dominates even out to much larger scales. To compare the large distance propagation differences of these two paradigms, we perform three-dimensional ideal magnetohydrodynamic adaptive mesh refinement simulations of both HD and PFD stellar jets formed via the same energy flux. We also compare how thermal energy losses and rotation of the jet base affects the stability in these jets. For the conditions described, we show that PFD and HD exhibit observationally distinguishable features: PFD jets are lighter, slower, and less stable than HD jets. Unlike HD jets, PFD jets develop current-driven instabilities that are exacerbated as cooling and rotation increase, resulting in jets that are clumpier than those in the HD limit. Our PFD jet simulations also resemble the magnetic towers that have been recently created in laboratory astrophysical jet experiments.
\end{abstract}

Key words: ISM: jets and outflows - methods: numerical - stars: magnetic field - stars: winds, outflows

Online-only material: color figures

\section{INTRODUCTION}

Non-relativistic jets are observed in the vicinities of many protostellar objects, young stellar objects (YSOs), and post-asymptotic giant branch stars. Plausible models suggest that jets are launched and collimated by a symbiosis of accretion, rotation, and magnetic mechanisms, which occur at the jet "central engine" (see Pudritz et al. 2007, for a review). The jet material must be accelerated to velocities beyond the escape speed and magnetically mediated launch models are often favored because they can provide the needed directed momentum (see Livio 2004; Pudritz 2004 for reviews).

Astrophysical jets are expected to be Poynting flux dominated (PFD) close to their engine. It is however unclear how far from the launch region the Poynting flux continues to dominate over kinetic energy flux, or whether the jets eventually become essentially hydrodynamic (Blackman 2007). The difference between these two possibilities is a difference between two magnetically launched outflow classes: (1) magneto-centrifugal jets (Blandford \& Payne 1982; Ouyed \& Pudritz 1997; Blackman et al. 2001; Mohamed \& Podsiadlowski 2007), in which magnetic fields only dominate out to the Alfvén radius, or (2) PFD magnetic tower jets (Shibata \& Uchida 1986; Lynden-Bell 1996; Ustyugova et al. 2000; Lovelace et al. 2002; Nakamura \& Meier 2004), in which magnetic fields dominate the jet structure, acting as a magnetic piston over very large distances from the engine. Indeed, magnetic fields with initially poloidal (radial and vertical) dominant geometries anchored to accretion disks have been shown to form tall, highly wound and helical magnetic structures, or magnetic towers, that expand vertically when laterally supported in pressure equilibrium with the ambient gas (Shibata \& Uchida 1986; Lynden-Bell 1996, 2003).
PFD jets carry large electric currents which generate strong, tightly wound helical magnetic fields around the jet axis. Simulations of such jets have found that magnetic fields play a role in the formation of current-driven kink instabilities and the stabilization of Kelvin-Helmholtz (KH) modes (e.g., see Nakamura $\&$ Meier 2004). However, while the correlation between the mechanical power of astrophysical jets and their main observable features (e.g., length, velocity, cocoon geometry, etc.) has been widely studied for kinetic-energy-dominated jets, this is not the case for PFD magnetic tower jets.

Recently, magnetized jets have been produced in laboratory experiments. These flows appear to exhibit key aspects of magnetic tower evolution (Lebedev et al. 2005; Suzuki-Vidal et al. 2010). In these experiments, performed on Pulsed Power current generators, the local injection of purely toroidal magnetic energy produced high Mach number $(\sim 20)$, fully radiative and fully magnetized jets. These magnetic towers exhibit PFD cavities with $\beta<1$ (where $\beta$ is the ratio of thermal to magnetic pressures) which expand supersonically into an unmagnetized ambient medium. Within the cavity, a central jet forms via hoop stresses. While the body of these jets has $\beta<1$, their core is a high $\beta$, kinetic-energy-dominated flow. The central jet evolution of these experiments also showed the growth of current-driven, nonlinear instabilities, in particular the kink, $m=1$, mode. As a result, the laboratory jets are eventually corrugated and become a collimated chain of magnetized "clumps" (Lebedev et al. 2005). These experiments were then modeled via resistive magnetohydrodynamic (MHD) simulations specifically developed for laboratory studies (Ciardi et al. 2007), where the details of the flow, including current distributions, were followed. The breakup of the jet into a sequence of collimated clumps has been suggested as an explanation for clumpy flows observed in YSO 
outflow systems (Hartigan \& Morse 2007; Yirak et al. 2010; Hartigan et al. 2011).

We note that the effect of plasma cooling via optically thin radiation has not been followed before in simulations of magnetic tower jets. Studies of weakly magnetized, kineticenergy-dominated jets show that this type of cooling can make the flow more susceptible to instabilities, such as $\mathrm{KH}$ modes (Hardee \& Stone 1997, and references therein). Recently, Ohsuga et al. (2009) studied magneto-centrifugally launched (MCL) jets with two-dimensional radiation-MHD simulations. Ohsuga et al. found that the strength of radiative cooling, which they control by changing the plasma density, affects the structure and evolution of both accretion disks and their associate jets. Although we follow thermal energy losses in the present study, we do not compute radiate transfer.

In this paper, we study the effects that thermal energy losses and rotation, independently of one another, have on the stability of PFD magnetic towers. For comparison we also run simulations of collimated asymptotically hydrodynamically dominated (HD) jets. Such HD jets could represent the asymptotic propagation regimes of MCL flows, which are distinct from PFD ones in that the latter remain dominated by magnetic flux out to much larger distances. Our comparison allows us to articulate how PDF flows differ from their hydrodynamic counterparts.

This paper is organized as follows: in Section 2, we describe the methodology and numerical code that we use for this study as well as our implementation of the gas, the velocity, and the magnetic field. The results of our simulations are presented in Section 3, where we follow the evolution, structure, and stability of our model jets. In Section 4, we discuss the implications of our simulations and how they compare with specific laboratory experiments and astronomical observations. Finally, we conclude in Section 5.

\section{MODEL AND INITIAL SETUP}

We model PFD and HD jets numerically by solving the equations of ideal (i.e., no explicit microphysical diffusivities) MHD in three dimensions. In non-dimensional conservative form these are given by

$$
\begin{gathered}
\frac{\partial \rho}{\partial t}+\nabla \cdot(\rho \mathbf{V})=\dot{\rho}_{\mathrm{inj}} \\
\frac{\partial(\rho \mathbf{V})}{\partial t}+\nabla \cdot\left(\rho \mathbf{V} \mathbf{V}+p \hat{\mathbf{I}}+\left(B^{2} / 2\right) \hat{\mathbf{I}}-\mathbf{B B}\right)=\dot{\mathbf{P}}_{\mathrm{inj}} \\
\frac{\partial E}{\partial t}+\nabla \cdot\left[\left(E+p+B^{2} / 2\right) \mathbf{V}-\mathbf{B}(\mathbf{V} \cdot \mathbf{B})\right]=\dot{E}_{\mathrm{inj}}-\Delta(T) \\
\frac{\partial \mathbf{B}}{\partial t}-\nabla \times(\mathbf{V} \times \mathbf{B})=\dot{\mathbf{B}}_{\mathrm{inj}},
\end{gathered}
$$

where $\rho, p, \mathbf{V}, \mathbf{B}$ and $\hat{\mathbf{I}}$ are the gas density, thermal pressure, flow velocity, magnetic field, and the unitary tensor, respectively. In Equation (3), $E=p /(\gamma-1)+\rho V^{2} / 2+B^{2} / 2$ and represents the total energy density whereas $\gamma$ is the ratio of specific heats. We have implemented source terms in the right-hand side of Equations (1)-(4) to account for the injection of mass, momentum, total energy, and magnetic flux. Since the cross sectional area of the jet base is fixed, these injections are respectively accomplished by injecting a mass density per unit time $\dot{\rho}_{\text {inj }}$, a momentum flux, $\dot{\mathbf{P}}_{\text {inj }}$, total energy flux minus radiation loss $\dot{E}_{\mathrm{inj}}-\Delta(T)$, and magnetic field per unit time $\dot{\mathbf{B}}_{\mathrm{inj}}$.
We solve these equations using the adaptive mesh refinement (AMR) numerical code AstroBEAR2. $0^{6}$ which uses the single step, second-order accurate, shock capturing CTU+CT (Gardiner \& Stone 2008) scheme (Cunningham et al. 2009; Carroll-Nellenback et al. 2011). While AstroBEAR2.0 is able to compute several microphysical processes, such as gas selfgravity and heat conduction, we do not consider these in the present study.

Our computational domain is defined within $|x|,|y| \leqslant$ $160 \mathrm{AU}$ and $0 \leqslant z \leqslant 400 \mathrm{AU}$, where $20 \mathrm{AU}$ is equivalent to one computational length unit. We use a coarse grid of $64 \times$ $64 \times 80$ cells plus two levels of AMR refinement which attain an effective resolution of 1.25 AU. Outflow boundary conditions were set at the left and right domain faces of both $x$ and $y$, as well as in the upper $z$ face. At the lower $z$ face, we combine two boundary conditions: reflective in those cells located at $\sqrt{x^{2}+y^{2}} \geqslant r_{e}$ and magnetic/jet source term values in those cells located at smaller radii, $r_{e}=31.4 \mathrm{AU}$. The latter represents the characteristic radius of the energy injection region, equal to the jets' radius, which is resolved by 24 cells.

We use BlueGene $/ \mathrm{P}^{7}$ an IBM massively parallel processing supercomputer of the Center for Integrated Research Computing of the University of Rochester, to run simulations for about 1 day using 512 processors.

\subsection{Initial Conditions}

We initialize our simulations with a static gas which has a uniform density of 200 particles $\mathrm{cm}^{-3}$ and a temperature of $10,000 \mathrm{~K}$. Gas is modeled with an ideal gas equation of state and a ratio of specific heats of $\gamma=5 / 3$. Magnetic fields are placed in a central cylinder of equal radius and height $r_{e}$. In cylindrical coordinates the magnetic vector potential is given by

$$
\mathbf{A}(r, z)= \begin{cases}\frac{r}{4}[\cos (2 r)+1][\cos (2 z)+1] \hat{\phi} & \\ \quad+\frac{\alpha}{8}[\cos (2 r)+1][\cos (2 z)+1] \hat{k}, & \text { for } r, z<r_{e} \\ 0, & \text { for } r, z \geqslant r_{e}\end{cases}
$$

where the parameter $\alpha$ has units of length and determines the ratio of toroidal to poloidal magnetic fluxes. We use $\alpha=$ 40 (computational length units) which is an arbitrary choice, yet consistent with the wound helical magnetic configurations expected from accretion disks (e.g., Blandford \& Payne 1982; Lynden-Bell 1996; Li et al. 2006) and produced in high energy density laboratory experiments of magnetic towers (Lebedev et al. 2005; Ciardi et al. 2007). Our choice of $\mathbf{A}$ is in part motivated by the work of Li et al. (2006). However, in our model, $\mathbf{A}$ is strictly localized to the central part of the grid.

We obtain the initial magnetic field, $\mathbf{B}^{\text {init }}$, by taking the curl of A:

$$
\begin{aligned}
& B_{r}^{\text {init }}=-\frac{\partial}{\partial z}\left(A_{\phi}\right)=2 r \cos ^{2}(r) \cos (z) \sin (z), \\
& B_{\phi}^{\text {init }}=-\frac{\partial}{\partial r}\left(A_{z}\right)=\alpha \cos ^{2}(z) \cos (r) \sin (r), \\
& B_{z}^{\text {init }}=\frac{1}{r} \frac{\partial}{\partial r}\left(r A_{\phi}\right)=2 \cos ^{2}(z)\left[\cos ^{2}(r)-r \cos (r) \sin (r)\right] .
\end{aligned}
$$

\footnotetext{
6 https://clover.pas.rochester.edu/trac/astrobear/wiki

7 https://www.rochester.edu/its/web/wiki/crc/index.php/Blue_Gene/P
} 



(a) Field components as a function of $r$ (left column) and $z$ (right column).

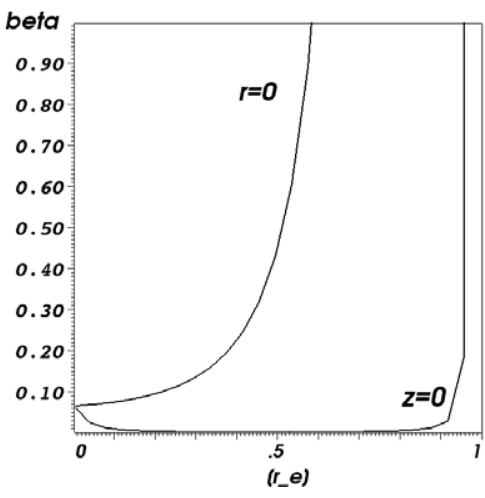

(b) Plasma $\beta$ (thermal to magnetic pressure ratio).

Figure 1. Magnetic field initial conditions. The field has a helical structure which is dominated by the toroidal component.

The magnetic field is normalized so that the initial thermal to magnetic pressure ratio $\beta$ is less than unity for $r<r_{e}$ and unity at $r=r_{e}$. In Figure 1, we show profiles of the initial magnetic field components (top and middle rows) and $\beta$ (bottom panel) as a function of the distance from the origin.

\subsection{Energy Injection}

We model jets by continually injecting energy into the central region of the grid, where $r, z \leqslant r_{e}$. Because one of the key goals of this work is to compare the observable propagation signatures of PFD jets versus HD ones (e.g., their length, velocity, density distribution), we inject either pure magnetic energy for the PFD case, hence the name magnetic towers, or pure kinetic energy for the HD case. We will now give details about the implementation of the jets.

\subsubsection{Magnetic Towers}

For these simulations, we inject magnetic flux by adding the initial magnetic field configuration (6) to the instantaneous central magnetic fields, $\mathbf{B}^{n}$, i.e.,

$$
\mathbf{B}^{n+1}=\mathbf{B}^{n}+\dot{\mathbf{B}}_{\text {inj }} d t,
$$

where $\mathbf{B}^{n+1}$ represents the central magnetic fields $\left(r, z \leqslant r_{e}\right)$ corresponding to the next computational time step, $d t$ is the current time step, and $\dot{\mathbf{B}}_{\text {inj }}=\mathbf{B}^{\text {init }} B_{c}$, where $B_{c}$ is the magnetic flux injection rate (see below).

For numerical stabilization, we also continually add static gas to the grid within $r, z<r_{e}$. This is accomplished using the expression

$$
\rho^{n+1}(r, z)=\rho^{n}(r, z)+\rho_{c}|\mathbf{B}(r, z)|^{2} d t,
$$

where $\rho^{n+1}(r, z)$ and $\rho^{n}(r, z)$ represent the gas densities corresponding to the next and the current time steps, respectively. We set the constant factor $\rho_{c}$ (which has units of $\mathrm{kg} \mathrm{m}^{-3} \mathrm{~s}^{-1} \mathrm{~T}^{-2}$ ) to be 0.01 computational units. Hence the average amount of injected gas is of order $0.001 \rho_{\text {amb }}^{0}$ per unit time, where $\rho_{\text {amb }}^{0}$ is the initial ( $t=0 \mathrm{yr})$ grid density of 200 particles $\mathrm{cm}^{-3}$; very dilute. Because of the factor $\left|B^{2}\right|$, the distribution of gas provided by (8) matches the gradients of magnetic pressure, thus we inject more gas at regions where the jet and magnetic cavity densities tend to be lower (Section 3.1).

\subsubsection{Hydrodynamical Jets}

For these simulations, we continuously inject kinetic energy and gas to the cells located at $r<r_{e}$ and $z<0$, i.e., within the bottom $z$ boundary. This region is equivalent to the base of the magnetic towers (discussed above). We impose constant boundary conditions in this region, based on the following three 
assumptions. (1) The collimation of the HD jet is presumed to have occurred at sub-resolution scales. (2) the HD jet is taken to have the same time averaged, maximum propagation speed as the PFD magnetic tower, that is,

$$
v_{j}=v_{z} \approx\left|\mathbf{B}_{\max }\right|\left(4 \pi \rho_{\mathrm{amb}}^{0}\right)^{-1 / 2} .
$$

(3) The injected energy fluxes of the HD and PFD magnetic tower jets are taken to be equal, i.e.,

$$
0.5 \rho_{j} v_{z}^{3} a=\left(|\mathbf{B}|^{2} / 8 \pi\right)\left(|\mathbf{B}|\left(4 \pi \rho_{\mathrm{amb}}^{0}\right)^{-1 / 2}\right) a,
$$

where $\rho_{j}$ is the jet's density and $a\left(=\pi r_{e}^{2}\right)$ is the area of the energy injection region. Hence,

$$
\rho_{j}=\left|\mathbf{B}_{\max }\right|^{2}\left(4 \pi v_{j}^{2}\right)^{-1}
$$

To ensure the condition (10) at all times, we set $B_{c}=10 /(1$ time computational unit) in Equation (7). We note that for the HD run, $\mathbf{B}=0$ everywhere and at all times, and that the values of $\mathbf{B}_{\max }$ and $\mathbf{B}$ in Equations (9)-(11) are taken from the magnetic tower simulation (above).

\subsection{Simulations}

We carry out six simulations: three magnetic tower runs and their corresponding hydrodynamical versions.

The adiabatic tower. This is a magnetic tower model which we have implemented as described in Section 2.2.1.

The cooling tower. This is a magnetic tower model which is identically to the adiabatic tower except for the addition of optically thin cooling which we have implemented using the tables of Dalgarno \& McCray (1972) via the source term $\Delta(T)$ in Equation (3).

The rotating tower. This is a magnetic tower model which is identical to the adiabatic tower except for the addition of a rotation profile at the jet base. This is accomplished by continually driving an azimuthal velocity to the central gas and frozen in magnetic fields of the tower. We use a velocity equal to the Keplerian speed corresponding to a two solar mass star. Specifically we impose

$$
v_{\phi}=\left\{\begin{array}{cl}
\sqrt{G 2 M_{\odot} / r}, & \text { for } r, z<r_{e} \\
0, & \text { for } r, z \geqslant r_{e}
\end{array}\right.
$$

Our choice of two solar masses is arbitrary but within the expected values for protostellar and YSO jet engines (e.g., see Konigl \& Pudritz 2000, and references therein). We note that the gas in our simulations is unaffected by gravitational forces, hence the centrifugal expansion produced by (12) is only balanced by magnetic pressure gradients. We do not expect significant dynamical differences with respect to a case in which gas was affected by gravity because we simulate jets far from the central star (Meier et al. 1997). Also, in our magnetic towers the magnetic fields are quite strong and the magnetic cavities contain very light gas (see below).

The HD jet. This is an adiabatic hydrodynamical jet model which we have implemented as described in Section 2.2.2.

The cooling HD jet. This is a hydrodynamical jet model which is identical to the HD jet except for the addition of the same thermal cooling source term that we use for the cooling tower run (above).

The rotating $H D$ jet. This is an adiabatic hydrodynamical jet model which is identical to the HD jet except for the addition of the base $\left(r<r_{e}\right.$ and $z$ within the bottom boundary of the computational domain) rotation profile described by Equation (12).

The structure and evolution of the HD, the cooling HD, and the rotating HD jet simulations are similar in terms of their global propagation characteristics (see Section 3.6). Hence, without loss of generality, in what follows we will only discuss about the adiabatic HD jet.

\section{RESULTS}

\subsection{Plasma Structure and Evolution}

In Figure 2, we show the evolution of the plasma with logarithmic false color particle density maps. From left to right, columns in the figure show the adiabatic, the rotating, and the cooling magnetic towers, and then the HD jet. Time increases downward by row. We denote the structures in the simulation as follows, based on the leftmost panel of row 2 (Figure 2): the jet core (white plasma within $r \lesssim 0.4$ ), the jet beam (lightestorange plasma within $r \lesssim 1.6$ ), the magnetic cavity (darkorange plasma within $1.6 \lesssim r \lesssim 4$, outside the jet), and the contact discontinuity $(\mathrm{CD}$, thin surface between the magnetic cavity and the swept up external medium). Beyond the CD we see the (light-orange) shocked ambient plasma.

The simulations show that the initial helically wound magnetic field launches PFD jets via magnetic pressure gradients: the low- $\beta$, low-density cavities expand via the $z$-gradient of the toroidal magnetic pressure between the tower and ambient medium. Inside the cavity, a central jet beam forms collimated by hoop stresses of the toroidal field (Section 3.2). The field in the cavity is in turn radially collimated by the pressure of the external high- $\beta$ plasma. The jets and their corresponding magnetic cavities expand and accelerate, especially along the $z$-axis. This drives bow shocks on the external unmagnetized media. This magnetic tower evolution is consistent with the analytical model of Lynden-Bell (1996, 2003), as well as with previous simulations of PFD jets and magnetic towers (see, e.g., Shibata \& Uchida 1986; Nakamura \& Meier 2004; Li et al. 2006).

Comparison of PFD magnetic towers with the HD jet reveals the following characteristics (Figure 2). The towers propagate with very similar vertical velocities but decelerate, by about $20 \%$, relative to the HD jet. This results because although the towers and the hydro jet have the same injected energy flux, the towers produce not only axial but radial expansion. The precollimated HD jet can only expand radially via a much lower thermal pressure. Thus all of the energy flux in the hydro-case for our setup is more efficiently directed to axial mechanical power. Moreover, the towers and the hydro jet show different structures: towers have a thin central jet which is susceptible to instabilities, whereas the HD jet's beam is thicker, smoother, and stable. We consistently see lower densities in the PFD tower cavities than in that of the HD case. The laboratory experiment magnetic towers of Lebedev et al. (2005) and Suzuki-Vidal et al. (2010) also show a magnetic cavity mostly void of plasma. The gas distribution inside the cavities shows more complex and smaller scale structures in the magnetic tower cases than in the HD one.

We see that the magnetic towers are affected by either cooling or rotation after their early expansion phase. Instabilities develop in their jet beam after $\sim 70 \mathrm{yr}$ (Section 3.5). The cocoon geometry of the cooling case (third column from left to right in Figure 2) is the fattest. We find that the volume of the ambient 

$\log (n)\left[\mathrm{cm}^{-3}\right]$
-7 .
0.
2.
3.
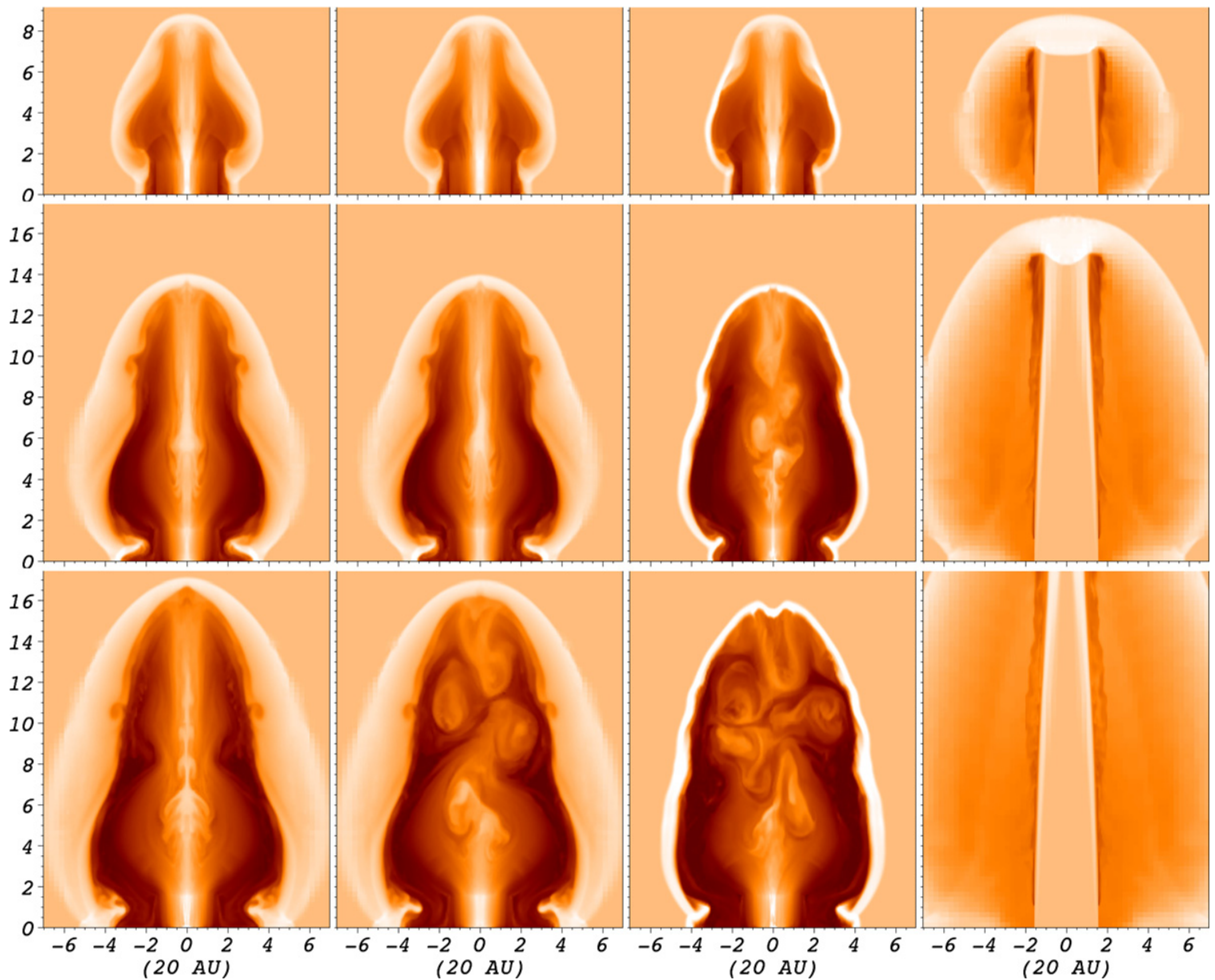


column), and the cooling (3rd column) cases, and the HD jet structure (4th column). From top to bottom time is equal to 42 , 84 , and 118 yr.

(A color version of this figure is available in the online journal.)

region which is affected by the towers is smaller in the cooling case, as expected (e.g., see Frank et al. 1998; Huarte-Espinosa et al. 2011). The above findings imply very different emission distributions for PFD- and HD-dominated jets. Future studies should address the creation of synthetic observations to assess these differences.

The evolution of the magnetic towers' gas density is consistent with that of their compressive MHD and hydrodynamic waves and shocks. In Figure 3, we show profiles of the relevant velocities of the towers $\left(v_{x}, v_{y}, v_{z}\right.$, the sound speed, and the Alfvén speed) along the jet axis, $r=0$, as a function of cooling, base rotation, and time. During their early stable propagation phase, the jet cores are mostly sub-Alfvénic and trans-sonic, independent of cooling or rotation. Fast-forward (FF) compressive MHD and hydrodynamic bow shocks are evident in the ambient medium, ahead of the jet heads.

Some evolutionary features are worth noting. From Figure 3, we see the FF shocks steepen in time (compare top to middle and middle to bottom rows). The swept of shells of unmagnetized ambient medium become relatively thin when radiative cooling is included (right column: compare top to middle and middle to bottom rows). The adiabatic and rotating cases show regions within the lower half of the jet where the flow speed is super-Alfvénic. Such regions are bounded by the reverse and the forward slow modes of compressive MHD waves, and characterized by high thermal to magnetic pressure ratios (Section 3.4).

At $t \gtrsim 90 \mathrm{yr}$ the distribution of waves and shocks of both the rotating and cooling cases (bottom row, middle and right columns) is significantly affected. This is due to the growth of nonlinear current-driven instabilities (Section 3.5). Possibly, pressure driven modes coexist with the current-driven ones in regions of high $\beta$. We see fast, though mostly subAlfvénic, azimuthal velocities, in the central parts of these jet cores.

To clarify the effect of cooling on our magnetic towers, we present temperature maps in Figure 4 below. We model radiation losses using (3), where $\Delta T \propto \rho^{2} \Lambda(T)$ and $\Lambda(T)$ is taken from the tables of Dalgarno \& McCray (1972). Figures 2 and 4 help to form a complete picture of the cooling strength. In the noncooling cases we see shocked ambient medium at temperatures of $T \sim 10^{5} \mathrm{~K}$. This material forms an extended shell surrounding the magnetic cavity formed by inflowing Poynting flux. In the cooling case, this shocked shell of ambient gas has cooled 

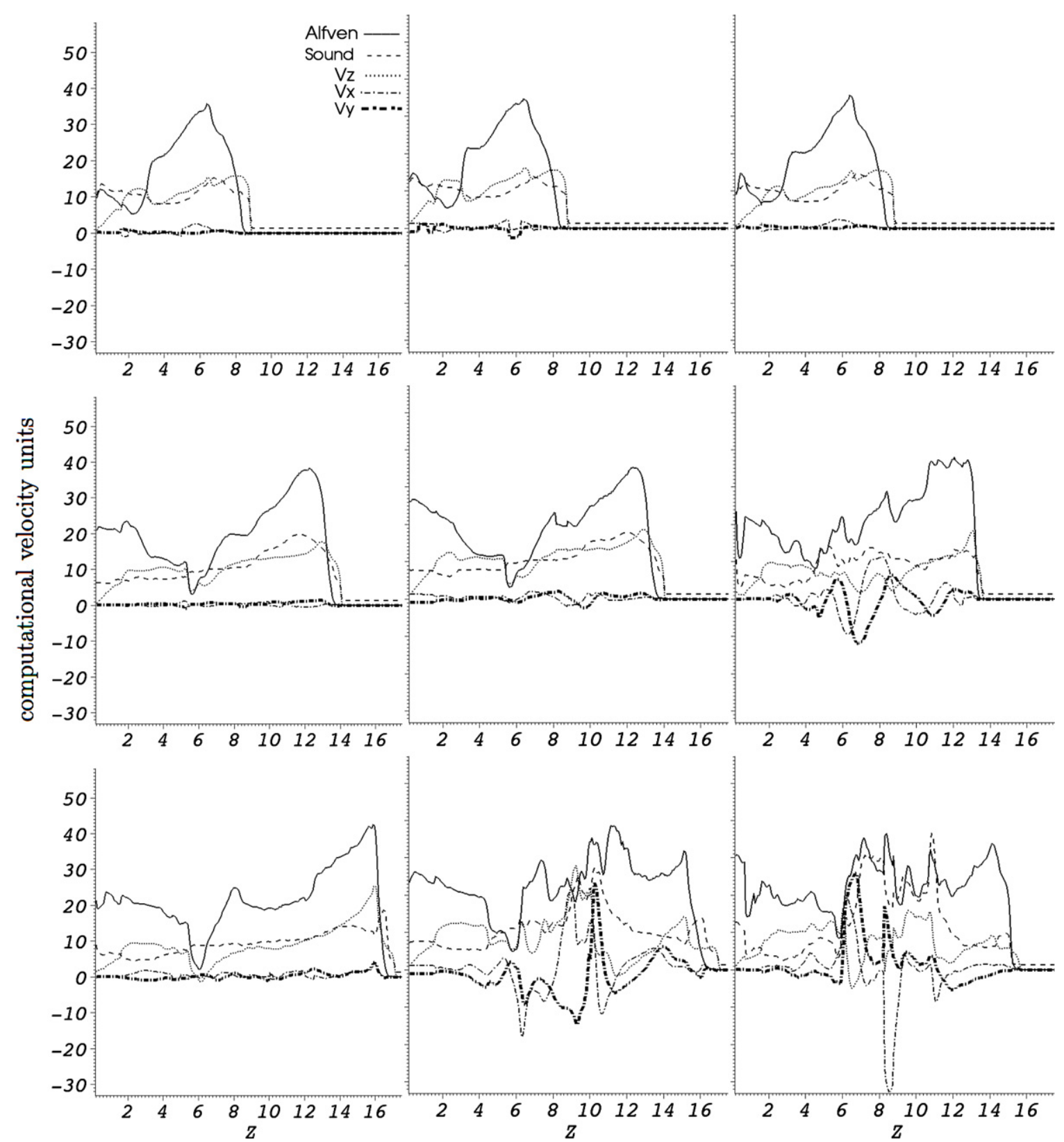

Figure 3. Evolution of the plasma velocities along the jets' axis. These are the magnetic towers in the adiabatic (left), the rotating (middle), and the cooling (right) cases. From top to bottom time is equal to 42, 84, and $118 \mathrm{yr}$. Each computational velocity unit is equivalent to $9.1 \mathrm{~km} \mathrm{~s}^{-1}$.

significantly to temperatures of $T \leqslant 10^{4} \mathrm{~K}$. The cooling has decreased the pressure in this region on the shell now becomes both thin and dense. Note we also see low temperature via cooling occur in the jet beam and the knots that form once the beam becomes unstable.

\subsection{Magnetic Fields and Current Density}

In Figure 5, we show the distribution of the towers' magnetic fields on the plane that contains the jets' axis. These are linear color maps of the absolute value of the toroidal to poloidal field component ratio. From left to right we show the adiabatic, the rotating, and the cooling cases, respectively, and time increases downward with row. We see that the magnetic flux changes sign along the radial direction. In general there are four main nested surfaces or layers of magnetic field lines (e.g., see middle row, left column panel): at the very core we see predominately poloidal (vertical $B_{z}$ ) fields surrounded by a surface of primarily toroidal (azimuthal $B_{\phi}$ ) flux. These field components represent the central core of the jet plasma column. They are collimated by two outer magnetic surfaces. The smaller of these two is dominated by poloidal lines, whereas the larger one is dominated by toroidal lines. These outer field lines are collimated by the thermal pressure and inertia of the external media.

As expected, the geometry of the towers' magnetic fields changes in time. Initially, the field lines have a highly wound helical configuration (Section 2.1). The magnetic pressure is very high and unbalanced in the vertical direction. The toroidal field lines thus move away from each other and the magnetic towers rise. The injection of magnetic flux sustains a non-forcefree configuration at the base of the tower. "New" field lines push the "old" ones upward then. The latter stretch and expand radially, making way for, and collimating, the jets' new field lines. 




Figure 4. Evolution of the towers' temperature. These logarithmic color maps show the magnetic towers in the adiabatic (left), the rotating (middle), and the cooling (right) cases. From top to bottom time is equal to 42,84 , and $118 \mathrm{yr}$.

(A color version of this figure is available in the online journal.)

After the towers' early expansion phase $(t \gtrsim 90 \mathrm{yr})$, we find, in agreement with the results of the previous section, that the jets of both the cooling and the rotating cases are affected by instabilities (Section 3.5). The final magnetic structure of the towers is clearest in the field line maps of Figure 6. These are the lines in the central part $\left(r \lesssim 1.2 r_{e}\right)$ of the adiabatic (left), the rotating (middle), and the cooling (right) towers at $t=118 \mathrm{yr}$. The top and bottom panels show the towers edge-on and pole-on, respectively. The adiabatic case shows quite ordered helical field lines and the strongest jet fields (red color) of all the towers. We also see that toroidal field lines tend to pile up at the tower's tip. Such a concentration of lines causes acceleration of the plasma at the tip of the adiabatic jet to supersonic speeds (see bottom, left panel in Figure $3, z \approx 15$ ). In contrast, the cooling tower (right panel) shows the weakest and most disordered field lines.
The middle and right panels show clear differences between the cooling and the rotating cases. The instabilities that develop in these towers are clearer in the rotating case (middle column; Section 3.5).

The magnetic fields are ultimately sustained by electric currents. In Figure 7, we show the evolution of the axial current density, $J_{z}$ (panels in this figure are arranged as in Figure 5). As expected we see a clear correlation between the distributions of the axial current density and the magnetic field. The jets carry a high axial current (red region) which is contained within a current-free region (white one) at larger radii. The main part of the return current (blue region) moves along the contact surface of the towers' cavity. This forms a closed circulation current system which is consistent with previous simulations of PFD jets (see, e.g., Lind et al. 1989; Lery \& Frank 2000; Nakamura 


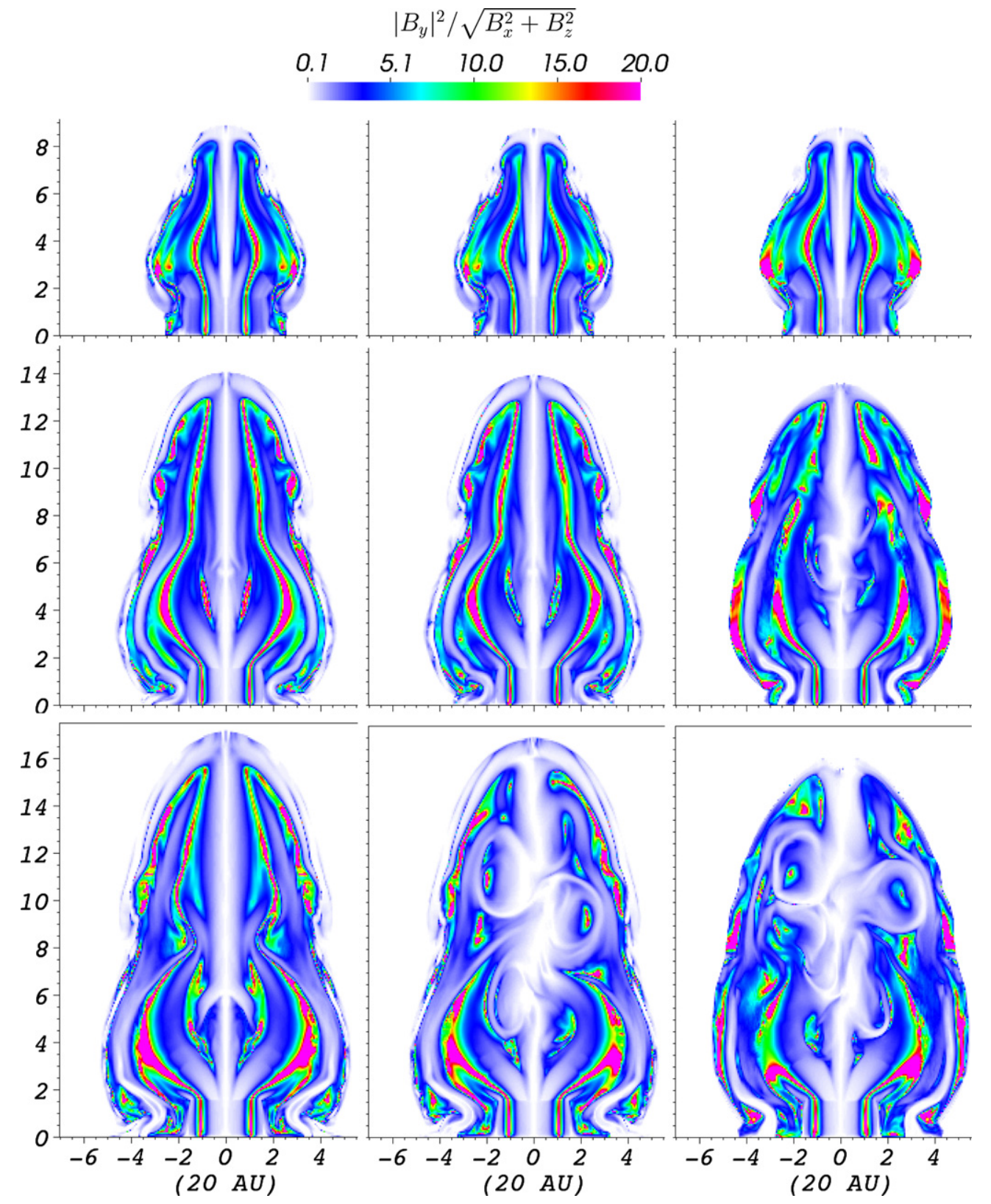

Figure 5. Evolution of the towers' magnetic fields. This is the ratio of the toroidal component over the poloidal one. $B_{y}=B_{\text {phi }}$ and is perpendicular to the maps. These linear color maps show the magnetic towers in the adiabatic (left), the rotating (middle), and the cooling (right) cases. From top to bottom time is equal to 42 , 84, and 118 yr.

(A color version of this figure is available in the online journal.)

\& Meier 2004; Ciardi et al. 2007) and the magnetic tower laboratory experiments of Lebedev et al. (2005) and SuzukiVidal et al. (2010). We note however that both the magnetic field and the current density are strictly localized in our model, i.e., no components of the current in the external medium. This is a characteristic feature of magnetic towers.

We see that the current $J_{z}$ is also affected by the instabilities that develop in the rotating and cooling towers after their early expansion phase (bottom row, middle and right columns). The effect of instabilities is most pronounced in the jet beam. As the jet breaks up into clumps the current becomes more localized. Numerical reconnection allows some of the sections of tangled fields to become isolated, however, the overall flow of axial current density continues as does the outer sheath of return current.

\subsection{Energy Flux}

To study the relative magnetic versus kinetic energy content of our magnetic towers we compute the Poynting flux, $f_{P}$, and the kinetic flux, $f_{k}$, defined respectively as

$$
\begin{aligned}
f_{P} & =\int_{s}[\mathbf{B} \times(\mathbf{V} \times \mathbf{B})]_{z} d S, \\
f_{k} & =\int_{s} \frac{1}{2} \rho|\mathbf{V}|^{2} V_{z} d S .
\end{aligned}
$$

The above integrals are taken over the area of the jet beams.

In Figure 8 we show logarithmic color maps of the distribution of the jet Poynting to kinetic flux ratio, $\log |Q(\mathbf{x}, t)|$, where $Q(\mathbf{x}, t)=f_{P} / f_{k}$, as a function of cooling, jet base rotation, and time. The maps show that only the core of the jets is dominated 


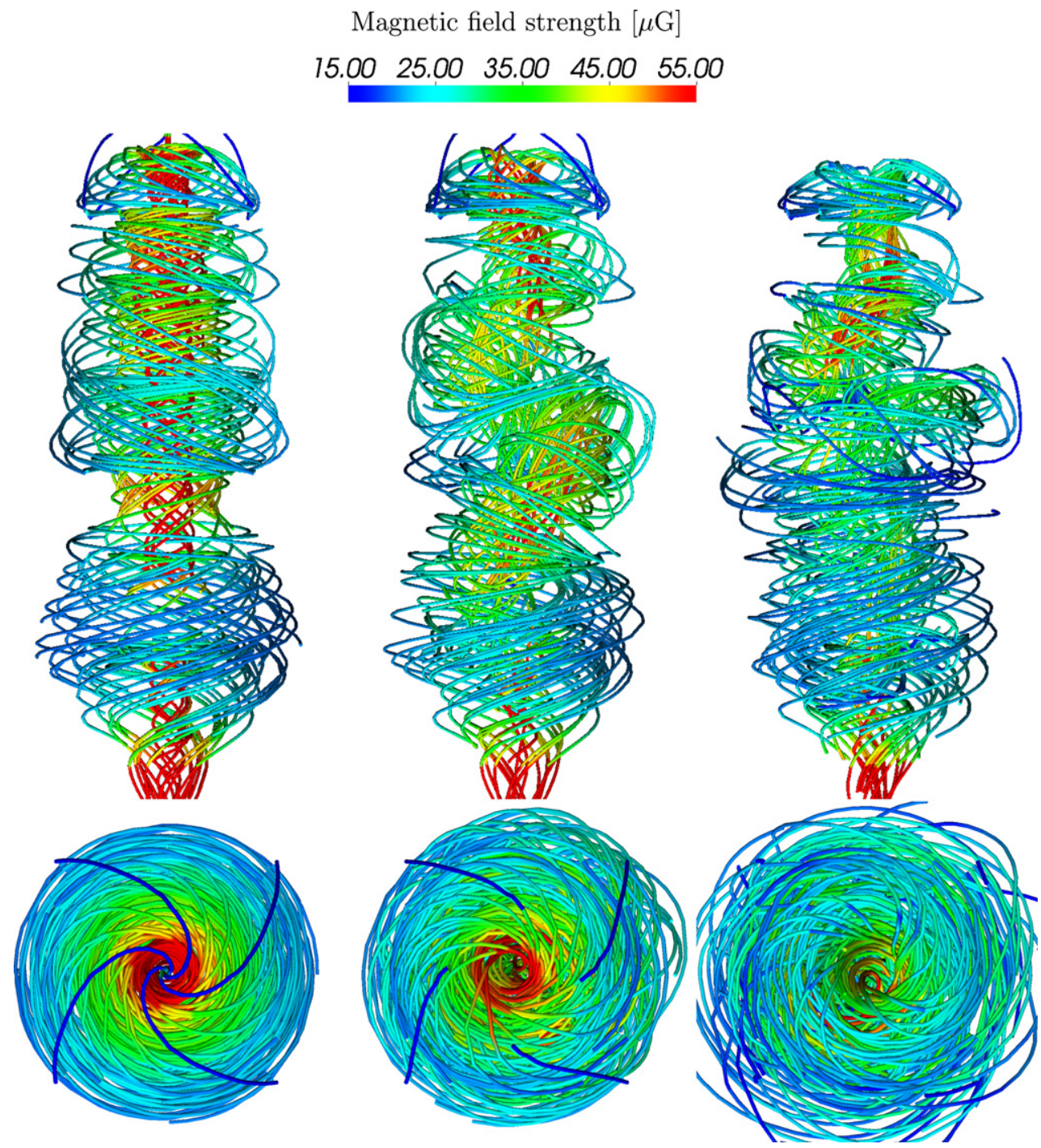

Figure 6. Central $\left(r \lesssim 1.2 r_{e}\right)$ magnetic field lines at $t=118 \mathrm{yr}$. From left to right these are the adiabatic, the rotating, and the cooling magnetic towers, respectively. Bottom panels show an upper view, pole-on. Open field lines are a visualization effect.

(A color version of this figure is available in the online journal.)

by kinetic energy flux $(Q<1$, blue region) while the bulk of the beam is $\operatorname{PFD}(Q>1$, red region) for all the cases (i.e., adiabatic, rotating, and cooling). This distribution is consistent with that found in the laboratory jets of Lebedev et al. (2005, Section 4). We confirm that our magnetic towers are indeed PFD. We note that the dark red stripes in Figure 8 correspond to regions where the toroidal field components are particularly strong (see Figure 5). To stress and clarify this point we also show logarithmic maps of $f_{k}$ (left panels) and $f_{P}$ (right panels) in Figure 9.

We find that the time average mean $Q$ of our magnetic tower beams - averaged over the adiabatic, cooling, and rotating cases-is $\sim 6$. This is about $2 / 3$ of the time average mean $Q$ in the magnetic towers simulated by Kato et al. (2004, see their Figure 3(b), bottom). We note that the spatial distribution of $Q$ in both our and their simulations is not isotropic and time dependent. Early in the evolution of our towers $Q$ is axisymmetric however the growth of the kink instabilities eventually leads to the development to far more spatial variability in $Q(x, y, z)$.

Our simulations show that the ratio of Poynting flux to kinetic energy flux is always greater than unity for the magnetic tower
$(Q>1)$. This should be compared with the models of jets created by MCL processes. While MCL jets begin with $Q>1$ on scales less than the Alfvén radius, in the asymptotic limit the kinetic energy flux comes to dominate the flux electromagnetic energy leading. Simulations of MCL launching in which the flow is cold and gas pressure can be ignored show typical values of $Q \sim 0.7$ at observationally resolved distances from the engine (Krasnopolsky et al. 1999, 2003). We leave a detailed comparison of PDF and MCL jets for the future.

\subsection{Forces}

Magnetic towers expand due to a combination of magnetic, thermal, and inertial forces. In Figure 10, we show the thermal to magnetic pressure ratio, $\beta$, using logarithmic gray-scale maps (arranged as in Figure 5). We find that $\beta$ for the magnetic towers is generally and consistently well below unity.

The adiabatic and rotating cases (left and middle columns, respectively) do show regions where $\beta>1$ close to $(r, z)=$ $(0,6)$. Such regions are located between the reverse and forward slow-mode compressive MHD waves (Figure 3, left and middle columns), and filled with subsonic, weakly magnetized plasma. 

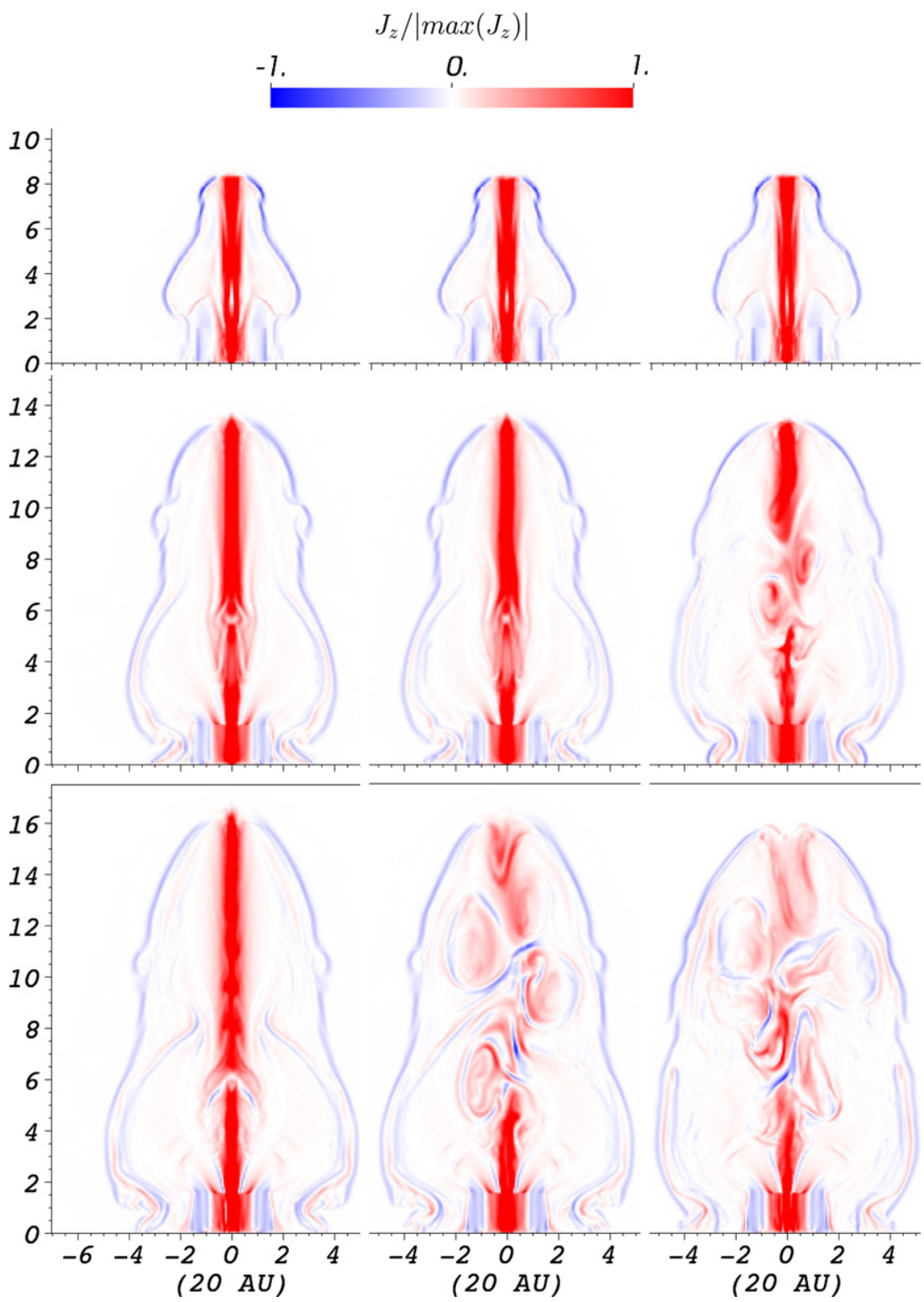

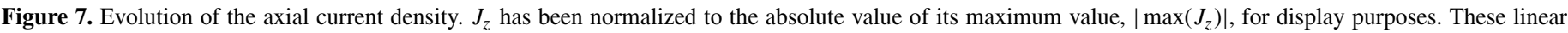

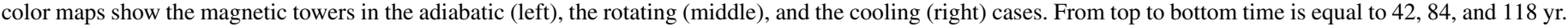

(A color version of this figure is available in the online journal.)

This high- $\beta$ region is strongly affected by cooling (right column) which reduces the thermal energy (see also Figure 4). Hence the total pressure of the surrounding plasma becomes further dominated by the magnetic component, and it collapses yielding higher compression ratios than the adiabatic case. The field in the cooling case also takes on a configuration amenable to instability. Thus, the plasma in the high- $\beta$ jet-core region plays a critical role on the overall stably of PFD outflows.

In Figure 11, we show the radial component of the forces in the magnetic towers during their intermediate evolutionary phase. From small to large radii these linear color maps show the jet core (dark colored regions), the jet beam edge (light colored regions), the cavities' central force-free region (white region), the $\mathrm{CD}$ (light colors), and finally the swept-up ambient gas (outer-most light features, bottom row) of the towers. In general, these figures show that the inward Lorentz force (top panels) is slightly stronger than both the inertial (or specific centrifugal $\left.v_{\perp}^{2} / x\right)$ and the thermal pressure, $(\nabla P)_{x}$, forces which push plasma outward. This fact is consistent with the results of Takeuchi et al. (2010) and Ohsuga \& Mineshige (2011).

Figures 7 and 10 show the character of the force density distribution responsible for confining the jets and their cavities. The jets are self-confined in the current-free region located at a few jet radii from the core (i.e., hoop stress). At larger radii, near the towers' contact surface, which is also the return current surface (blue outer region in Figure 7), the magnetic pressure is weak and thus it only requires a mild ambient pressure (lightgray outer region, Figure 10) to confine the outer part of the towers.

In Section 3.1, we saw that the magnetic towers decelerate with respect to the HD jet. This can be understood with the bottom panel in Figure 11 where we see that the magnetic flux that is injected into the towers (within model $r \lesssim 1.5$; Section 2.2.1) causes not only axial $(z)$ expansion, but also radial expansion via magnetic pressure. In contrast, the kinetic energy flux in the HD jet (not shown) is overwhelmingly axial. 


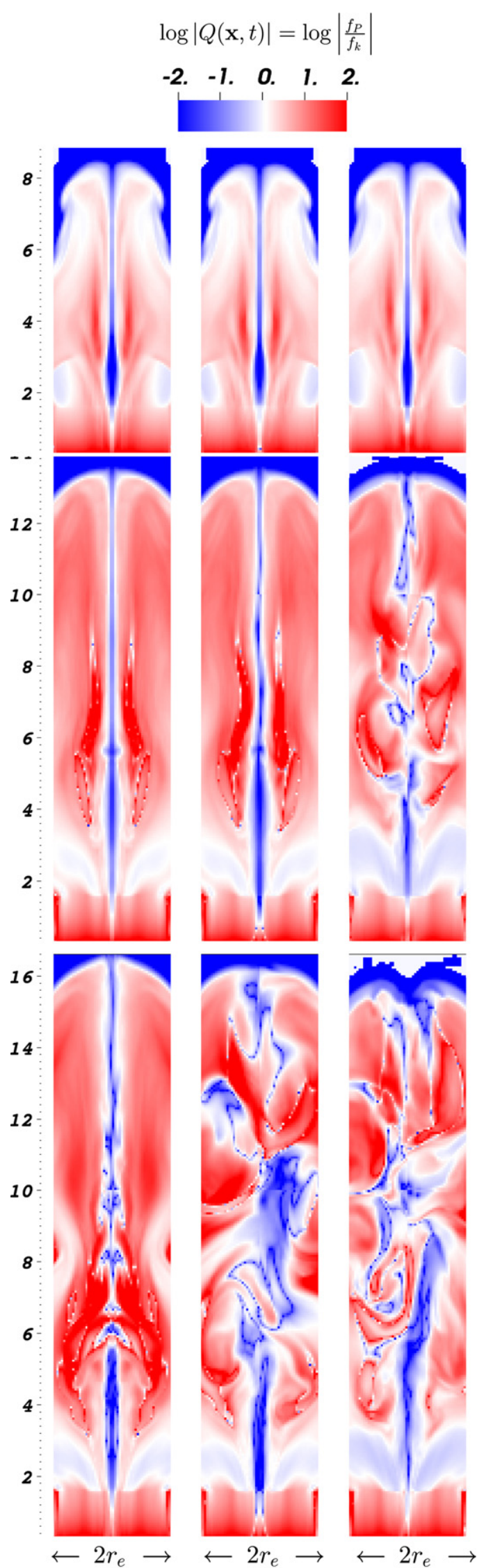

Figure 8. Distribution and evolution of the jet beam Poynting to kinetic flux ratio. These logarithmic maps show the jets of the magnetic towers in the adiabatic (left), the rotating (middle), and the cooling (right) cases. From top to bottom time is equal to 42,84 , and $118 \mathrm{yr}$.

(A color version of this figure is available in the online journal.)

\subsection{Stability}

The structure and expansion of our PFD magnetic tower jets are affected by current-driven instabilities. We see evidence of the pinch, $m=0$, the kink, $m=1$, and the $m=2$ normal mode perturbations. These are expected in expanding magnetized plasma columns and consistent with the models of Nakamura \& Meier (2004, and references therein) and Ciardi et al. (2007)—but see also Song \& Cao (1983) —and also with the laboratory experiments of Lebedev et al. (2005) and SuzukiVidal et al. (2010). We find that the kink perturbations grow and lead to instabilities in the cooling tower, first, and later also in the rotating tower.

Perturbations with modes $m=0$ and 2 develop in the adiabatic jet after expanding for $\sim 80 \mathrm{yr}(\sim 70 \%$ of the total simulation running time). These are caused by radial gradients in the magnetic fields located within the jet beam, at the boundary of the current-free, force-free region. The thermal and magnetic components of the total pressure balance each others' perturbations locally. As a result, the core of the adiabatic jet becomes a helical column with an elliptical cross-section. The growth rate of these $m=0$ and $m=2$ perturbations is $>120 \mathrm{yr}$, which is longer than the simulation final time. Thus, we are seeing only the linear develop of the modes. Finally we note resemblances (Figure 2, bottom left panel) to structures seen in the $\mathrm{S}_{\mathrm{II}}$ emission distribution of the jet in HH 34 (Reipurth et al. 2002 , and references therein).

The central part of the towers' jet beams is high- $\beta$ plasma columns where $\left|B_{\phi} / B_{z}\right| \ll 1$ (Figure $5, B_{\phi}=B_{y}$ ). To understand their development, we can appeal to standard Kruskal-Shafranov criterion for the kink instability, namely (Boyd \& Sanderson 2003)

$$
\left|\frac{B_{\phi}}{B_{z}}\right|>\left|\left(\beta_{z}-1\right) k r_{\text {jet }}\right|
$$

where $\beta_{z}=2 \mu_{0} P / B_{z}^{2}$ and $k^{-1}$ is the characteristic wavelength of the current-driven perturbations. In Figure 3 (right column), we see that the cooling jet's core shows $\beta_{z} \sim 1(z \sim 4-5$ at time $=84 \mathrm{yr}$ and $z \sim 6-11$ at time $=118 \mathrm{yr})$. This means that the cooling tower does not have sufficient thermal energy, in comparison with the adiabatic and rotating cases, to balance the magnetic pressure kink perturbations. This is consistent with what we see in the towers' density and temperature maps, Figures 2 and 4 . In addition, we see that the jet core radius of the cooling tower is about $20 \%$ smaller than that of the adiabatic tower. This is consistent with what is found in laboratory experiments of magnetized supersonic jets, in which outflows with different cooling rates are compared (A. Ciardi et al. 2012, in preparation). Both of these effects (thermal energy losses and core radial compression) reduce the right-hand side of Equation (14), making the system more susceptible to the growth of kink instabilities in the cooling tower.

For the rotating case, we find that rotation at the base of the jet beam, Equation (12), causes a progressive, slow amplification of the toroidal magnetic field component of the jet. This process is evident in the four panels at the bottom left of Figure 3, where we see that in general the Alfvén speed is higher in the rotating case (middle column) than in the adiabatic one (left column). This growth is likely sufficient to amplify the left-hand side of Equation (14) pushing the jet into the unstable regime. Since the field grows linearly with the differential rotation, the growth rate is likely proportional to the imposed amount of rotation. 


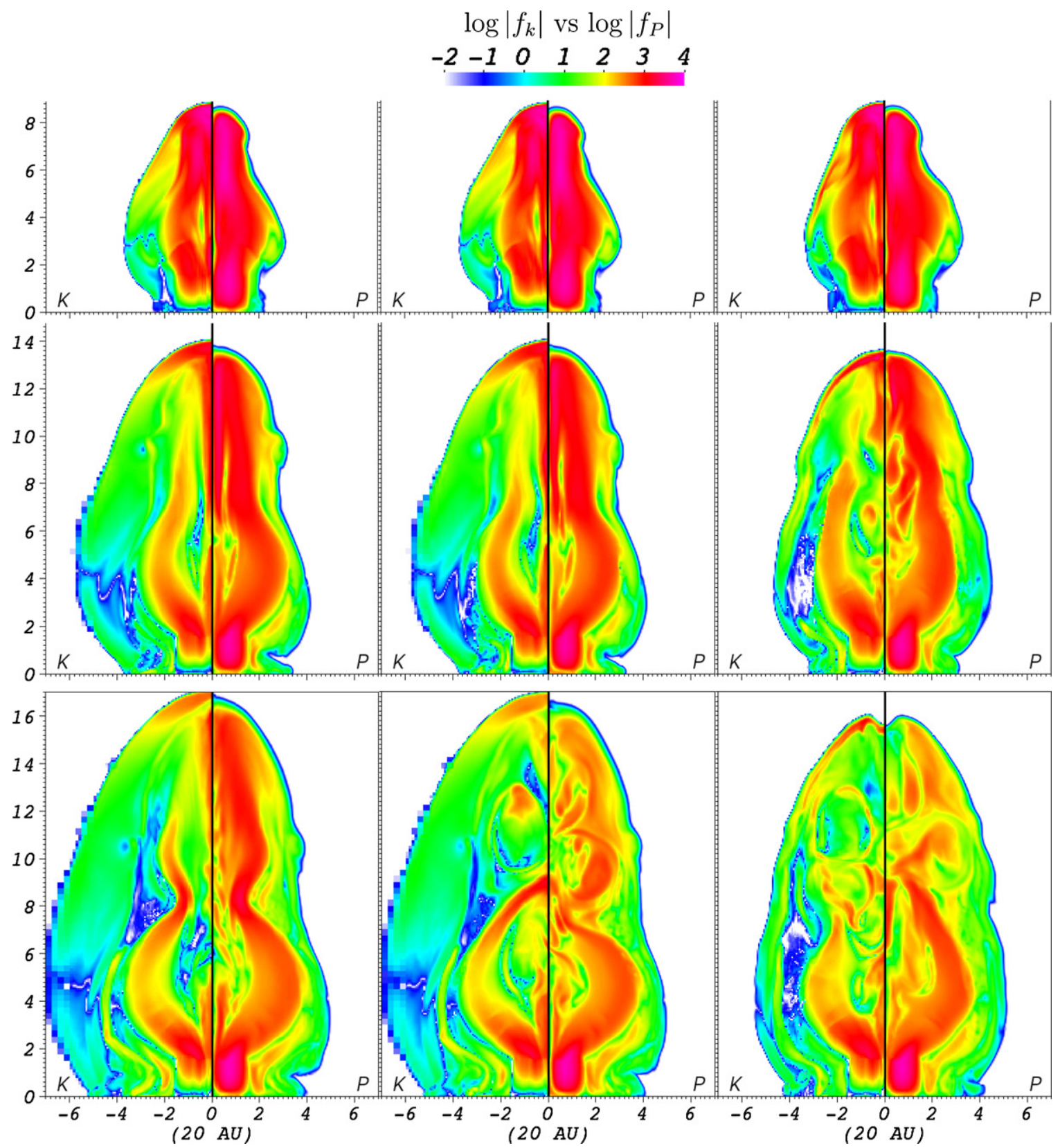

Figure 9. Distribution and evolution of the towers' kinetic energy (left) and Poynting (right) polar fluxes in computational units. These logarithmic maps show the magnetic towers in the adiabatic (left), the rotating (middle), and the cooling (right) cases. From top to bottom time is equal to 42,84 , and $118 \mathrm{yr}$.

(A color version of this figure is available in the online journal.)

We have not tested this as we have used only one value of the differential rotation.

Note that the towers are not completely destroyed even when unstable, and the amplitude of the kink perturbations in the jet is about twice its radius (Figure 6), in agreement with the Kruskal-Shafranov criterion (Kruskal et al. 1958; Shafranov 1958).

\subsection{The HD Cooling and HD Rotating Cases}

In addition to the four simulations presented above, we have carried out two variations of the HD jet run: one with cooling and one which has a base rotation profile which follows Equation (12), just as in the rotating magnetic tower run (Section 2.3). We found that the results of the cooling HD jet simulation were consistent, as expected, with those found in previous similar studies, i.e., thin jet-produced shocks with high compression factors (see, e.g., Frank et al. 1998; Hardee \& Stone 1997). For the regimes we have studied, the propagation and structure of the HD jets are affected by both cooling and rotation in ways which have been studied before and which do not alter the global propagation properties of the flow, i.e., no instabilities are introduced as in the magnetic tower case.

\section{IMPLICATIONS FOR JET OBSERVATIONS, EXPERIMENTS, AND FUTURE WORK}

The results of the simulations help guide our understanding of the evolution of PFD magnetic towers. In particular the simulations show new details of the cavity-jet connection, the 


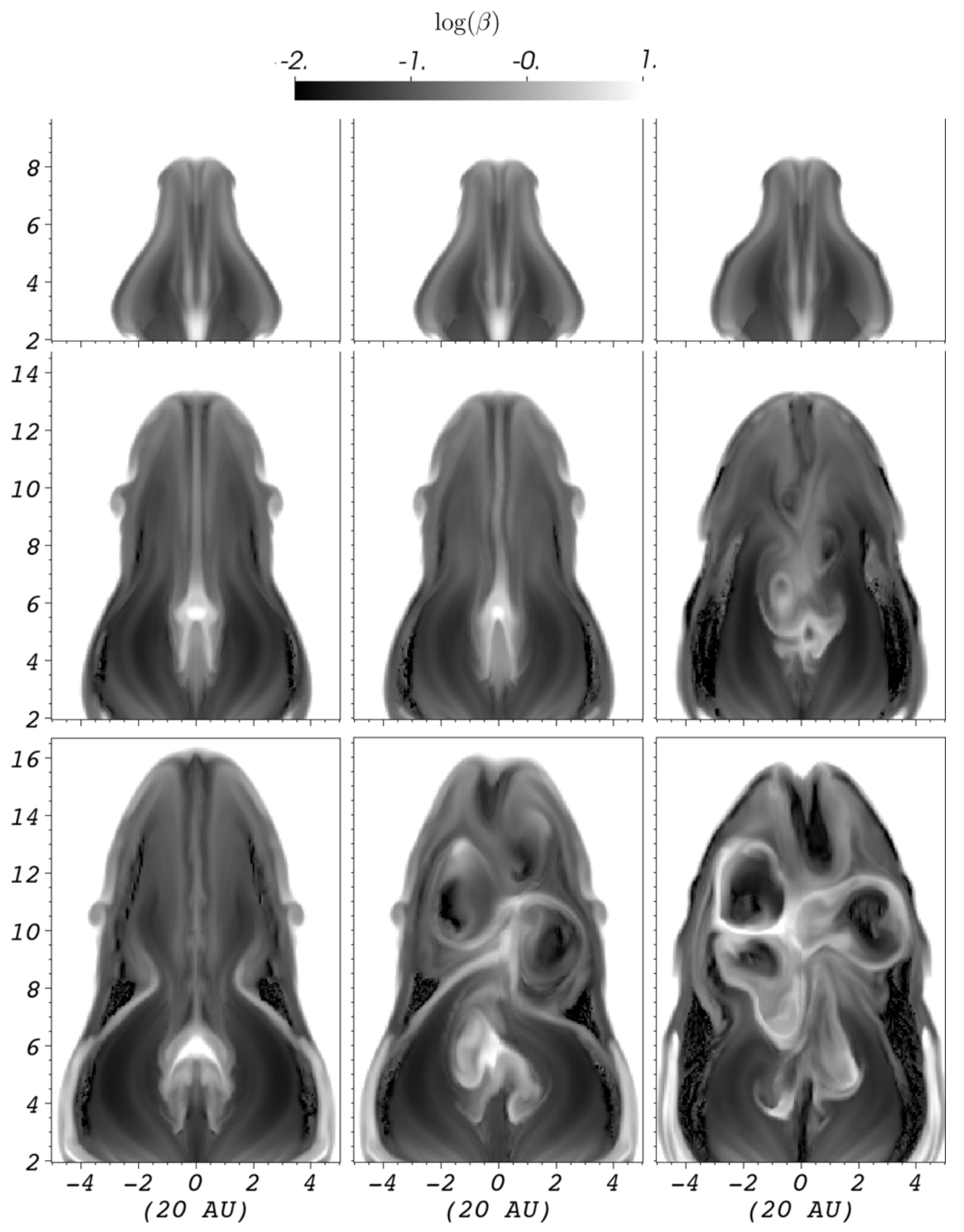

Figure 10. Evolution of the thermal to magnetic pressure ratio. These logarithmic gray-scale maps show the magnetic towers in the adiabatic (left), the rotating (middle), and the cooling (right) cases. From top to bottom time is equal to 42, 84, and $118 \mathrm{yr}$.

evolution of the tower given different assumptions (cooling, rotation, etc.) as well as providing some insight into the stability properties of the central jets which form in the flow.

For non-relativistic collimated flows magnetic towers have been proposed as mechanisms for launching some classes of YSO. While the flows downstream at observable distances $\left(>10^{3} \mathrm{AU}\right)$ seem to be kinetic energy dominated, at smaller scales a PFD region may be expected. As Hartigan et al. (2007) have shown, what few measurements of magnetic fields exist in YSO jets indicate there must be a region of sub-Alfvénic, PFD-dominated flow on scales of order 100 AU or less. In addition, these simulations demonstrate (and as laboratory experiments have shown) that the long-term evolution of magnetic towers may yield a series of collimated clumps whose magnetization properties vary over time. In this way, PFD flows may evolve into kinetic-energy-dominated jets at large distances from the central engine. Planetary nebula (PN) offer another potential application of non-relativistic PFD-dominated flows. Magnetic fields are already expected to play an important role in launching pre-planetary nebulae (PPNe) based on an observed mismatch between momentum in the PPN flow and momentum available through radiation. A number of papers have discussed how strong magnetic fields might create PPN or PN collimated flows (Blackman et al. 2001; Frank \& Blackman 2004; Matt et al. 2006). Observations of PPN and PN offer morphological similarities to the kinds of features seen in our simulations, such as hollow lobes and axial clumps. Future work might address these connections.

Of particular importance is the connection between the models presented in this paper and recent "laboratory astrophysics" 

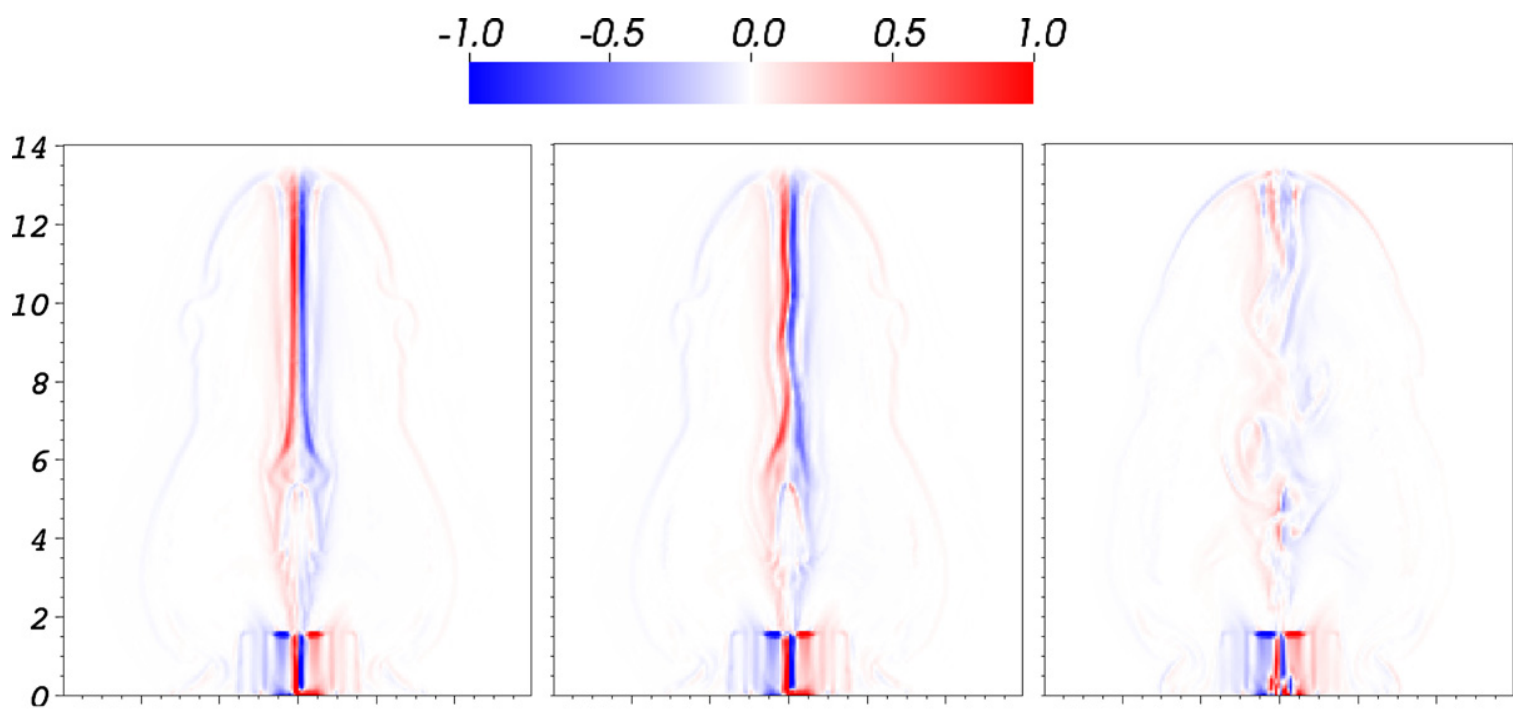

(a) Lorentz force, $(\mathbf{J} \times \mathbf{B})_{x} / F_{\max }$


(b) Inertial force minus the thermal pressure, $\left(v_{\perp}^{2} / x-(\nabla P)_{x}\right) / F_{\max }$

Figure 11. Radial forces at the intermediate evolutionary phase $(t=84 \mathrm{yr})$ of the towers. Forces are normalized to the maximum value, $F_{\max }$. The horizontal axis is $x=r$, the vertical axis is $z$, and $v_{\perp}$ is perpendicular to the maps.

(A color version of this figure is available in the online journal.)

experiments. These studies utilized Pulsed Power technologies and were successful in creating high Mach number, fully radiative, magnetized outflows (Lebedev et al. 2005; Ciardi et al. 2007, 2009; Ampleford et al. 2008; Frank et al. 2009). The outflows were created when TW electrical pulses (1 MA, $250 \mathrm{~ns})$ are applied to a radial array of fine metallic wires. Lorentz forces ablated plasma from the wires creating an ambient plasma above the array. After the complete ablation of wires near the central electrode, the current switches to the plasma and creates a magnetic cavity with a central jet (i.e., a magnetic tower). The central part of the jet is confined and accelerated by the pressure of the toroidal field. Return current flows along the walls of the magnetic cavity, which is in turn confined by the thermal pressure and by inertia of the ambient plasma. As the magnetic cavity expands, the jet becomes detached and propagates away from the source at $\sim 200 \mathrm{~km} \mathrm{~s}^{-1}$. Instabilities which resemble the kink mode $(m=1)$ develop within the body of these jets fragmenting them into well-collimated structures with characteristic axial non-uniformities.
Thus, the evolution of magnetic towers in the laboratory shows a range of features that are strikingly similar to what is seen in our simulations. This concordance is all the more noteworthy in that our initial conditions were in no way tuned to the experiments and are, in fact, a modified version of what can be found in a number of purely astrophysical studies (e.g., $\mathrm{Li}$ et al. 2006). Thus, it appears that the laboratory experiments and the simulations support each other, as well as the conclusion that both are revealing generic properties of PFD outflows.

While we did not study relativistic outflows, some aspects of the comparative behavior between HD and PFD jets revealed by our models might still apply. The fragmentation of the PFD magnetic tower core, for example, implies that rather than continuous jet beams we would expect high-resolution observations to reveal essentially "clumpy" jets with a distribution of velocities, densities, and magnetization. In this way our models, except the cooling ones, can be considered to articulate classes of flow features in active galactic nucleus radio jets (Tavecchio et al. 2003), X-ray binaries (Miller-Jones et al. 2007) and, perhaps, 
gamma-ray bursts (Morsony et al. 2010). But both relativistic and different radiative cooling generalizations are needed to confirm or refute the implications of our present calculations for such regimes.

There is opportunity for future work to focus more closely on the links with the laboratory experiments. In particular, issues related to the development of kink mode instabilities, their nonlinear resolution, and the evolution of clumpy magnetized jets should be explored more fully and in more detail.

Regarding the effect of rotation at the base of the jets on their stability, we note that Moll et al. (2008) have carried out three-dimensional simulations of magneto-centrifugally driven, conical jets and found that kink instabilities are stronger when a rigid rotation profile is imposed, in comparison to a Keplerian rotation profile. Rigid rotation seems to induce a shearless magnetic field (Moll et al. 2008). A direct comparison with our calculations must be made carefully though; our initial magnetic configuration has a dominant toroidal component and no radial component, while the initial field setup of Moll et al. (2008) is purely radial. Also, our rotating magnetic tower is continually affected by injection of magnetic flux, which is not the case of the conical jets of Moll et al. (2008).

\section{CONCLUSIONS}

We have carried out three-dimensional ideal MHD simulations of PFD- and HD-dominated jets to compare their structure and evolution subject to the same injected energy flux, and to study the effects of cooling and jet rotation on the jet stability. We note that our HD cases can, in principle, emulate asymptotic propagation regimes of MCL jets if those jets become kinetic energy dominated at large distances. Magnetic towers will however remain PFD at large radii.

Our simulations lead us to the following conclusions. Helical localized magnetic fields injected into a region of low pressure will launch PFD, magnetic towers via magnetic pressure gradients. Towers consist of a low-density low- $\beta$ plasma, the radial collimation of which is caused by the pressure of the external plasma. Within the towers a higher density, higher $\beta$ jet forms collimated by the magnetic field lines located within the cavity.

We found that PFD jets create structures that are more susceptible to instabilities relative to purely hydrodynamical jets given the same injected energy flux. Unstable modes in the magnetic towers differ according to conditions within the flow. The adiabatic PFD jet is unstable to $m=0$ and 2 mode perturbations, and its core adopts an elliptical cross-section. On the other hand, the PFD jet with a Keplerian rotating base exhibits an $m=1$ kink mode instability. The beam is not completely destroyed but adopts a chaotic clumpy structure. Base rotation causes a slow amplification of the toroidal field exacerbating a pressure unbalance in the jet's core that leads to instability. The cooling PFD jet also shows an $m=1$ kink mode instability. Cooling reduces the thermal energy of the jet's core, making the thermal pressure insufficient to damp the magnetic pressure kink perturbations. The cooling PFD beam shows the fastest growth rate of the kink instability.

Our magnetic tower (PDF jets) simulations are in good agreement with the laboratory experiments of Lebedev et al. (2005). In both our simulations and the experiments: (1) jets carry axial currents which return along the contact discontinuities, (2) the jet cores have a high $\beta$, (3) jet beams and cavities are PFD, (4) jets are eventually corrugated by current-driven instabilities becoming a collimated chain of magnetized "clumps" or "bullets." The similarity between our models and the experiments is particularly noteworthy because our implementation was not tuned to represent the laboratory results. This strengthens the case for the usefulness of laboratory experiments in articulating new features of astrophysical MHD flows in cases where similarity conditions can be obtained.

We found that PFD jets decelerate by about $20 \%$ relative to the HD ones given the same injected energy flux. This is because PFD jets produce not only axial but radial expansion due to magnetic pressure. All of the pre-collimated energy flux of the HD case is more efficiently directed to axial mechanical power. Also, the long-term evolution of PFD jets yield a series of collimated clumps, the magnetization properties of which may vary over time. PFD flows may thus eventually evolve into HD jets at large distances from the central engine.

Our work shows that outflows launched as magnetic towers show a different behavior compared with those launched by MCL mechanisms when the MCL flows become asymptotically kinetic energy flux dominated. As was shown by Hartigan et al. (2007), in YSO flows some mechanism may be needed to reduce the magnetization of plasma close to the jet source. If these flows begin as magnetic towers then the disruption of the central jets via kink modes may provide a means to produce collimated high- $\beta$ clumps of material as is observed in HH flows. Thus, our work may help to lead methods for distinguishing between different launch mechanisms by providing descriptions of asymptotic flow characteristics where observations might be possible.

Financial support for this project was provided by the Space Telescope Science Institute grants HST-AR-11251.01-A and HST-AR-12128.01-A, by the National Science Foundation under award AST-0807363, by the Department of Energy under award DE-SC0001063, and by Cornell University grant 41843-7012. S.L. acknowledges support from EPSRC Grant No. EP/G001324/1. We thank David Meier and Neil Turner for helpful discussions.

\section{REFERENCES}

Ampleford, D. J., Lebedev, S. V., Ciardi, A., et al. 2008, Phys. Rev. Lett., 100, 035001

Blackman, E. G. 2007, Ap\&SS, 307, 7

Blackman, E. G., Frank, A., \& Welch, C. 2001, ApJ, 546, 288

Blandford, R. D., \& Payne, D. G. 1982, MNRAS, 199, 883

Boyd, T. J. M., \& Sanderson, J. J. 2003, (Cambridge: Cambridge Univ. Press), 548

Carroll-Nellenback, J. J., Shroyer, B., Frank, A., \& Ding, C. 2011, arXiv: 1110.1616

Ciardi, A., Lebedev, S. V., Frank, A., et al. 2007, Phys. Plasmas, 14, 056501

Ciardi, A., Lebedev, S. V., Frank, A., et al. 2009, ApJ, 691, L147

Cunningham, A. J., Frank, A., Varnière, P., Mitran, S., \& Jones, T. W. 2009, ApJS, 182, 519

Dalgarno, A., \& McCray, R. A. 1972, ARA\&A, 10, 375

Frank, A., \& Blackman, E. G. 2004, ApJ, 614, 737

Frank, A., Ciardi, A., Yirak, K., \& Lebedev, S. 2009, RevMexAA: Conf. Ser. 36, 193

Frank, A., Ryu, D., Jones, T. W., \& Noriega-Crespo, A. 1998, ApJ, 494, L79

Gardiner, T. A., \& Stone, J. M. 2008, J. Comput. Phys., 227, 4123

Hardee, P. E., \& Stone, J. M. 1997, ApJ, 483, 121

Hartigan, P., Frank, A., Foster, J. M., et al. 2011, ApJ, 736, 29

Hartigan, P., Frank, A., Varniére, P., \& Blackman, E. G. 2007, ApJ, 661, 910

Hartigan, P., \& Morse, J. 2007, ApJ, 660, 426

Huarte-Espinosa, M., Frank, A., \& Blackman, E. 2011, in IAU Symp. 275, Jets at all Scales (Cambridge: Cambridge Univ. Press), 87

Kato, Y., Hayashi, M. R., \& Matsumoto, R. 2004, ApJ, 600, 338

Konigl, A., \& Pudritz, R. E. 2000, in Protostars Planets IV, ed. V. Mannings, A. P. Boss, \& S. S. Russell (Tucson, AZ: Univ. Arizona Press), 759

Krasnopolsky, R., Li, Z.-Y., \& Blandford, R. 1999, ApJ, 526, 631

Krasnopolsky, R., Li, Z.-Y., \& Blandford, R. D. 2003, ApJ, 595, 631 
Kruskal, M. D., Johnson, J. L., Gottlieb, M. B., \& Goldman, L. M. 1958, Phys. Fluids, 1, 421

Lery, T., \& Frank, A. 2000, ApJ, 533, 897

Lebedev, S. V., Ciardi, A., Ampleford, D. J., et al. 2005, MNRAS, 361, 97

Li, H., Lapenta, G., Finn, J. M., Li, S., \& Colgate, S. A. 2006, ApJ, 643, 92

Lind, K. R., Payne, D. G., Meier, D. L., \& Blandford, R. D. 1989, ApJ, 344,89

Livio, M. 2004, Bal. Astron., 13, 273

Lovelace, R. V. E., Li, H., Koldoba, A. V., Ustyugova, G. V., \& Romanova, M. M. 2002, ApJ, 572, 445

Lynden-Bell, D. 1996, MNRAS, 279, 389

Lynden-Bell, D. 2003, MNRAS, 341, 1360

Matt, S., Frank, A., \& Blackman, E. G. 2006, ApJ, 647, L45

Meier, D. L., Edgington, S., Godon, P., Payne, D. G., \& Lind, K. R. 1997, Nature, 388,350

Miller-Jones, J. C. A., Rupen, M. P., Fender, R. P., et al. 2007, MNRAS, 375 , 1087

Mohamed, S., \& Podsiadlowski, P. 2007, in ASP Conf. 372, 15th European Workshop on White Dwarfs, ed. R. Napiwotzki \& M. R. Burleigh (San Francisco, CA: ASP), 397

Moll, R., Spruit, H. C., \& Obergaulinger, M. 2008, A\&A, 492, 621
Morsony, B. J., Lazzati, D., \& Begelman, M. C. 2010, ApJ, 723, 267

Nakamura, M., \& Meier, D. L. 2004, ApJ, 617, 123

Ohsuga, K., \& Mineshige, S. 2011, ApJ, 736, 2

Ohsuga, K., Mineshige, S., Mori, M., \& Kato, Y. 2009, PASJ, 61, L7

Ouyed, R., \& Pudritz, R. E. 1997, ApJ, 482, 712

Pudritz, R. E. 2004, Ap\&SS, 292, 471

Pudritz, R. E., Ouyed, R., Fendt, C., \& Brandenburg, A. 2007, in Protostars Planets V, ed. B. Reipurth, D. Jewitt, \& K. Keil (Tucson, AZ: Univ. Arizona Press), 277

Reipurth, B., Heathcote, S., Morse, J., Hartigan, P., \& Bally, J. 2002, AJ, 123,362

Shafranov, V. D. 1958, Sov. J. Exp. Theor. Phys., 6, 545

Shibata, K., \& Uchida, Y. 1986, PASJ, 38, 631

Song, M.-T., \& Cao, T.-J. 1983, Chin. Astron. Astrophys., 7, 159

Suzuki-Vidal, F., Lebedev, S. V., Bland, S. N., et al. 2010, IEEE Trans. Plasma Sci., 38, 581

Takeuchi, S., Ohsuga, K., \& Mineshige, S. 2010, PASJ, 62, L43

Tavecchio, F., Ghisellini, G., \& Celotti, A. 2003, A\&A, 403, 83

Ustyugova, G. V., Lovelace, R. V. E., Romanova, M. M., Li, H., \& Colgate, S. A. 2000, ApJ, 541, L21

Yirak, K., Frank, A., \& Cunningham, A. J. 2010, ApJ, 722, 412 